\title{
La gerencia pública. Del "sistema de botín" a la gestión profesional
}

\author{
Ramón Antonio Quintín Medina*
}

\section{Resumen}

El trabajo tiene como objetivo estudiar las experiencias y tendencias de la Gerencia Pública en América Latina y Venezuela. Se examina el paradigma gerencial que ha predominado en las últimas décadas y se reconoce el surgimiento de un nuevo paradigma gerencial en sintonía con los cambios a nivel mundial. A partir de la realidad venezolana se exploran los factores institucionales que limitan el desarrollo del perfil deseable para el gerente público sustitutivo del "sistema de botín". Se utiliza como estrategia metodologica, el replanteo del contenido del concepto de Gerencia Pública y el análisis de los avances gerenciales en los países desarrollados y la experiencia exitosa de algunos países de América Latina.

Palabras claves: Gerencia pública, sistema del botín, gestión profesional, reforma administrativa, Venezuela.

\section{Public management. From the "buskin system" to the professional management}

\section{Abstract}

The article has as objective to study experiences and tendencies of Public Management in Latin America and Venezuela. It analyzes the management paradigm which has been used in the last decades and is recognizes the appearance of a new management paradigm, in concordance with world changes. It studies the institutional factors of the Venezuelan reality, factors that limit the development of the desired profile of the public

Recibido: 24-11-95. Aceptado: 29-01-96

- Postgrado en Administración Pública. Profesor de la Escuela de Administración de la Universidad Central de Venezuela. Asesor de numerosos proyectos de reforma en Venezuela y en otros palses de América Latina 
manager, a substitute of the "buskin system". The methodological strategy was the re-formulation of the concept of Public Management, the analysis of managerial progress in developed countries and the successful experiences of same latinoamerican countries. Key words: Public management, buskin system, professional management, administrative reform, Venezuela.

\section{¿Por qué la Gerencia Pública?}

Al comenzar la década de los ' 60 se inició en América Latina un intenso proceso de reforma de las administraciones públicas. Tales procesos se sustentaban en las concepciones económicas "estructuralistas" que se abrieron paso como consecuencia del fracaso de las políticas monetaristas experimentadas en la década anterior. La CEPAL y la Alianza para el Progreso dieron cuerpo al estructuralismo económico, perfilando una sólida corriente de pensamiento y de acción económica, política y social que fue acogida prácticamente por todos los gobiernos de la región, y erigida como una alternativa viable a los procesos de insurgencia política.

En esencia, la lógica de dicho modelo seguía la siguiente secuencia: la situación de subdesarrollo sólo podia superarse mediante un amplio proceso de industrialización; el Estado era el único agente con capacidad para emprender ese proceso; por tanto, era preciso un Estado fuerte y altamente interventor en la producción directa de bienes y servicios; paralelamente, debían emprenderse intensos esfuerzos de modernización de las estructuras tradicionales, con fuerto sello rural, en tanto facto de represamiento de los recursos de la sociedad (Rostow, Germany, etc.) La acción del Estado debía sustentarse en la "planifica- ción para el desarrollo económico y social", la cual, a su vez, sólo podía potenciarse mediante una administración pública con capacidad para formular $\theta$ instrumentar tales planes.

Se fue modelando, de esta manera, una política común de reformas para casi todo el continente; el lenguaje de los años ' 60 era extraordinariamente similar a partir del sur del Río Grande. Reforma agraria, reforma educativa, reforma impositjva, etc.; plenaban los discursos de intelectuales, partidos políticos y estadistas. Se consagra también, desde ese momento, la reforma administrativa o "administración para el desarrollo", como condición para llevar a cabo las transformaciones (ILPES), y obviamente Venezuela no estaba al margen de ese circuito continental.

Los primeros pasos de la reforma administrativa podian calificarse como de reduccionismo estructural. En efecto, los cambios se concentraron inicialmente en las estructuras administrativas, en los organigramas, en la dimensión formal de las organizaciones públicas. Se pensaba ingenuamente que las transformaciones en las estructuras administrativas, por si solas, podian dinamizar el funcionamiento del aparato público. Los primeros trabajos de la Comisión de Administración Püblica dan cuenta de esa estrategia $y_{i}$ por supuesto, de su fracaso.

La reflexión posterior se centró en 
la necesidad de tecnificar el funcionamiento de la Administración. Se llegó a asociar la modernidad con la tecnología, especialmente con aquella que habia sido suficientemente probada en los palses de origen. La transferencia de tecnologla administrativa acrítica fue una consecuencia perversa de la estrategia tecnificadora; tal es el caso del presupuesto por programas, entre otros muchos ejemplos.

A principios de la década de los 70 se inicia una estrategia centrada en el recurso humano: era preciso incrementar la base de conocimientos del funcionario público con el fin de garantizar el éxito de la tecnificación administrativa. Es la épo. ca del auge de las escuelas e institutos de capacitación de servidores públicos, con lejana referencia a la famosa Escuela $\mathrm{Na}$ cional de Administración Pública (ENA) de Francia y sustentados en aprendizajes eminentemente cognitivos. El deterioro progresivo de la Administración obligó a ampliar esa perspectiva hacia horizontes valorativos, ya que se sostuvo que el problema radicaba principalmente en las actitudes de los funcionarios en torno al trabajo. Sin embargo, no obstante el creciente y rico acervo de conocimientos e investigaciones que fueron desarroiladas alrededor del fenómeno de lo público, la Administración continuaba siendo percibida como un aparato ineficiente, rígido, sometido a intereses sectoriales, colonizado por los partidos políticos y carente de probidad.

La sinuosa y accidentada trayectoria del proceso reformista condujo finalmente a identificar un foco fundamental de atención: la gerencia pública.
A ella se le atribuyen inmensas potencialidades para dinamizar transformaciones en las múltiples dimensiones de la Administración Pública y, a la vez, para enfrentar los enormes desafios de la actual crisis nacional.

En efecto, se comparte absolutamente la idea del rol estratégico que cumplen los niveles directivos de la Administración Pública en el proceso de modernización del aparato gubernamental. $\mathrm{De}$ hecho, la desburocratización de la Administración, la calidad de los servicios que se prestan a los ciudadanos, la motivación y desarrollo del funcionario, la adopción de estructuras administrativas adecuadas, la instrumentación de tecnologías pertinentes y eficaces, la resolución de la compleja trama psicosocial de las organizaciones públicas, y tantos otros fenómenos organizacionales; son fundamentalmente procesos que están dentro de la esfera de atención de la gerencia pública y de su capacidad dependerá, sin duda, el éxito institucional. Por lo demás, asf lo revelan las "islas de excelencia" o las organizaciones de mayor eficiencia relativa que existen en el aparato gubernamental.

Cabe una reflexión final en este punto, aunque no es esta la oportunidad para desarrollarla: el escenario continental ha cambiado de actores y ya no es la misma representación; el Estado fuerte e interventor ha dado paso al mercado; de las grandes nacionalizaciones se ha pasado a las grandes ( $y$ no tan grandes) privatizaciones. Como consecuencia, el aparato estatal tiende a su reducción; se redimensionan las instituciones, y los criterios y sesgos empresariales tienden a 
invadir el campo de lo público. Incluso se enjuicia la capacidad intrínseca del Estado para administrar con probidad'. En efecto, en años recientes, el enfasis reformista de América Latina - y no menos en Venezuela- estuvo puesto en la dimensión económica y en la racionalización estructural del aparato administrativo. Se procuró determinar el ámbito de lo público y de lo privado y se concluyó en la necesidad de aumentar la eficiencia y eficacia del sector público. El eje de los análisis y propuestas se centró en la privatización, la liberalización y la apertura. El diagnóstico inicial de la crisis regional asignaba al excesivo crecimiento del Estado y al populismo económico un peso fundamental. En consecuencia, los procesos modernizadores recomendaban medidas tendientes a promover la estabilidad a través de los mecanismos del mercado y a reducir el tamaño del Estado y su grado de intervención en la economía.

El proceso de reforma implica, más bien, que el Estado debe asumir otras responsabilidades, además de la diferente ponderación que debe otorgar a sus funciones tradicionales. De hecho, la modernización del Estado forma parte de la modernización de la sociedad; es la respuesta trente a la crisis económica y está directamente asociada a los procesos de democratización política y social. La reforma del Estado venezolano requiere sumarse a los planteamientos que consideran que este esfuerzo es una exigencia para el logro de mayor equidad, concebida ésta como un valor en sí, pero también como un prerrequisito para un desarrollo sostenido, cuyo objetivo principal es aumentar el bienestar y elevar la calidad de vida de todas las personas. $Y$ en esta concepción, la gerencia del sector público juega un papel fundamental.

\section{La crisls de Identldad de la Gerencla Públlca}

En efecto, a la legitimidad legal sobre la cual se sustenta la actuación tanto del Estado como de la administración pública, se han añadido las exigencias de los valores de la equidad social y de la eficacia y la eficiencia en el uso de los recursos públicos. De hecho, la eficacia y eficiencia de la gestión pública se han convertido hoy en una condición básica no sólo para la reforma del Estado sino

\begin{abstract}
Esta situaclón ha servido de argumento, en muchos sectores, para Justfficar una vasta corriente privatizadora y el desarrollo de la ideologia de la "publlc cholse". Esta sefiala, en esencla, que cada individuo procura maximizar su proplo blenestar, y el mercado constluirla el instrumento más eficlente para el logro de este objetvo. Las preferenclas, en la forma en que ellas se manifiestan en el mercedo, estarlan a salvo de toda forma de coerción, en especial de las que podrian derivar de la institucionalidad politica. Esta última es visuallzada como un instrumento que los individuos usan para obtener ventajas a costa de sus semejantes, a través de alguna forma de coerción. Sólo ol mercado estarfa a salvo de la influencla contaminante de la politica.

Las autoridades politicas y los burdcratas, de acuerdo con este enfoque, tenderian a usar las instituclones públicas para maximizar su proplo blenestar. Para atenuar estas distorsiones es necesario reducir lo más posible el papel de las entidades públlcas y dejar que la mayor parte de las decislones sean adoptadas all donde existe "libertad para elegir". Ese instancla no es otra que el mercado. De hecho, el neoliberalismo ha tomado este argumento a partir del enfoque de la "opclón pública" desarrollado en Estados Unidos, en la forma en que ha sido expuesto por J. Buchanan, G. Tullock y otros.
\end{abstract}


también para el logro de la competitividad y del propio desarrollo económico.

En estas circunstancias, aparece en escena el concepto y la práctica de la gerencia pública. Sin embargo, muy tempranamente la gerencia pública comienza a introducir severas confusiones en el campo de la administración del Estado, y ella misma pugna por adquirir sustantividad disciplinaria y profesional. Por un lado, se la ha intentado reducir a un mero apéndice del Derecho Administrativo y de la Ciencia Politica. Por el otro, se la ha sesgado incorporándole mecánicamente los principios y técnicas del management empresarial, bajo entorno privado, a la gestión de bienes y servicios públicos, bajo entorno de no mercado y con fuerte componente de "autoridad".

Ello ha tendido a generar ciertos excesos, pues la reivindicación, por ejemplo, de mayor libertad de actuación para los directivos públicos, con el argumento de igualar la discrecionalidad de los gestores privados, ha producido la anulación de los viejos $\theta$ inútiles sistemas de control, pero sin sustituirlos por un nuevo y eficiente sistema de rendición de cuentas $y$ de responsabilidades, incrementando asi tanto la arbitrariedad del poder como el potencial de corrupción.

Por su parte, el Derecho Administrativo -sostiene Joan Prats- (1995:92) se ha mostrado incapaz para entender y establecer un diálogo fecundo con la gerencia pública, lo que por lo demás no tiene nada de extraño, dado el acartonamiento de la corporación administrativista en el paradigma "antidiscrecional". En efecto, señala Prats, el Derecho Administrativo se ha plasmado en un paradigma que atiende y resuelve los problemas de legitimidad del Estado Liberal de Derecho, pero ha sido irrelevante e indiferente al desarrollo del Estado Social y Democrático, y resulta ajeno, cuando no contradictorio, con el desarrollo de la racionalidad gerencial pretendida por el management de hoy. "El Derecho Administrativo, todavía dominante, se encuentra aprisionado en un paradigma que no responde ya a la dinámica de los hechos, que conduce a la estéril negación y crítica de los mismos y que impide el diálogo fecundo con el management público, en el marco renovado de unas Ciencias Administrativas, hoy más necesarias que nunca, habida cuen. ta de las rápidas y profundas transformaciones a que está siendo sometido el sector público de nuestro tiempo".

En términos generales, el dilema de nuestra Administración Pública se mueve en un doble marco de exigencias polares aparentemente contradictorias: 0 incorporamos el management con la expectativa de una gestión más eficiente de los asuntos públicos, y con los riesgos de desatar el Leviathan, o contenemos al Estado sobre la base de la legitimidad legal que le imprime el Derecho Administrativo, pero reducido a la clásica función de administrar el régimen de libertades.

\section{La crisis del paradigma gerenclal}

- Se está en presencia de una nueva realidad de las organizaciones en general, lo que implica la necesidad de adoptar posturas actualizadas en torno a la función directiva. De hecho, el contexto de incertidumbre ha generado fuertes ruidos 
en las organizaciones, sumidas a tasas de cambio acelerado y afectadas por un gran número de variables, agotando con ello la capacidad de predicción. Se terminó la época de aprender de la realidad -se sostiene en el Massachusetts Institute of Tecnology (M.I.T.) Se requiere un "aprendizaje generativo" (The Economist, 1989). En este mismo sentido, $Y$. Dror refiere que un gerente está condenado al fracaso si le ha ido bien en el pasado. Todo ello complica el management: su curriculum, su perfil, $y$, en definitiva, genera crisis en el paradigma mismo de la función gerencial.

Hasta hace poco tiempo, el exito gerencial se le vinculaba al clásico POSDCORB de Gulick" . Gerente "ideal" serla el que manejara con toda eficiencia las funciones del "proceso administrativo", vista desde una perspectiva formalista. Serla básicamente el gerente capaz de manejar la organización formal. La teoría gerencial moderna está en una etapa absolutamente diferente. Investigaciones como las de Henry Mintzberg (1975) en Canadá, que revelaron sistemáticamente la actividad real de los gerentes exitosos, llegan a la conclusión de que tienen como grandes funciones: la relación interpersonal, el manejo de información y la de decidir, que involucra componentes importantes de negociación institucional, y que el POSDCORB es un aspecto menor de su labor.

Otra de las investigaciones difundidas sobre esta materia es la de John Kotter, de la Universidad de Harvard, quien evidenció que los ejecutivos de más altos logros son aquellos que sostienen una intensa relación "cara a cara" con los demás, en un clima no burocrático. Este particular estilo les permite: 1) armar una adecuada agenda de decisiones, ya que les facilita identificar en tiempo real las cuestiones verdaderamente estratégicas para la organización; 2) lograr que se hagan las cosas a través de un grupo grande y diverso de personas; 3) crear una red de relaciones sobre las que se apoya su capacidad real de instrumentación. Así las agendas y las redes colocan a estos gerentes en buena posición para responder al flujo de eventos y de cambios. Otros autores confirman los hallazgos de Kotter en el sentido de que la condición de éxito gerencial descansa en la velocidad de reacción al entorno y lograr, a su vez, identificar correctamente la agenda estratégica real para enfrentar los problemas clave. Por el contrario, el culto a la documentación y al papeleo es el factor más perjudicial para una gestión exitosa.

Hay un aspecto de singular relevancia que conspira contra el despliegue de las capacidades gerenciales, y muy especialmente en las organizaciones públicas, aunque constituye a la vez un desaffo de enormes proporciones para quienes ejercen la función directiva: la cultura predominante en torno a la concepción de las estructuras organlzaclonales, que suelen configurarse bajo criterios de rigidez, de formalismo y de sólidas jerarquilas.

2 De las siglas en inglés Planning, Organising, Staffing, Directing, Cordinating, Reporting y Budgeting. 
Por el contrario, éstas deben ser adecuadas para actuar en un mundo de incertidumbre. La organización debe maximizar su flexibilidad. Edgar Schein sostiene al respecto la necesidad de terminar con la idea de la jerarquía y de las organizaciones jerárquicas. Se dibujan organizaciones con tendencia cada vez mayor a configurar redes. Según el M.I.T., las organizaciones más competitivas se fundamentan en redes, por lo que les denomina "organizaciones hologramas", donde cada parte de la organización tiene suficiente información para reconstituir el todo; están ligadas horizontalmente; la descentralización es más aguda; etc. Sin embargo, el tema de las estructuras organizacionales está, en este momento, en una etapa de experimentación.

Por otro lado, se ha impuesto en los últimos años el concepto de las organ-. zaclones que aprenden, desarrollado por Peter Senge. Se trata de que la organización aprenda en tiempo real y simultáneo de los problemas que plantea la incertidumbre del contexto. En tal sentido, se ha sostenido que administrar no es simplemente "dingir recursos humanos hacia metas", sino más bien "permitir que los individuos respondan creativamente ante situaciones cambiantes", lo que incluye la idea de aprendizaje. En ese mismo contexto, se afirma que el gerente debe ser preparado como un buen legitimador de la realidad, es decir, debe interpretar 10 que sucede en su entomo $y$ transmitirlo a la organización. Por lo tanto, el gerente es el primero en la IInea de aprendizaje, para saber cuáles son los hechos importantes que deben ser transmitidos a la organización y al mismo tiem- po saber evaluar las tendencias. De hecho, las organizaciones deben convertirse en laboratorios de aprendizaje.

Lo mencionado anteriormente no es un campo ajeno a la gerencia pública. En un mundo cada vez más competitivo el papel del gerenté público será más exigente y complejo que en el pasado. El Estado está en un proceso de transformación fundamental, lo cual requiere de una inteligencia que favorezca la competitividad y permita enfrentar los gigantescos desaflos contemporáneos. Preparar un agente público para un Estado inteligente y fuerte, y una sociedad civil igualmente fuerte para enfrentar los desafíos de esta magnitud, implica estar muy abierto a las fronteras tecnologicas de la gerencia contemporánea y participar en la revolución de los paradigmas gerenciales que vive el mundo.

\section{La situación de la Gerencla Públlca en Venezuela}

La población de gerentes públicos del nivel medio y superior del pais alcanza aproximadamente a 40.000 efectivos, incluyendo las tres instancias territoriales. Estos ejecutivos, en su gran mayoría, distan considerablemente del perfil deseable de gerente público y de un marco de condiciones apropiadas para el despliegue de sus potencialidades. Las pocas "islas de excelencia" que existen en el sector público lo son precisamente por la atención que han puesto al recurso gerencial y, particularmente, a su profesionalización. Por el contrario, la ineficiencia institucional tiene una estrecha relación con la ausencia de una política guberna- 
mental en esta materia; ausencia que se manifiesta en los siguientes rasgos. (COPRE, 1994:263-266)

- El ingreso a los cargos de Gerencia Media y Alta no requiere requisitos de capacidad profesional:

- No están definidas las fuentes de reclutamiento; gran parte de estos cargos son cubiertos de fuentes externas.

- No existe un organismo orientado a la formación de gerentes públicos que siva como fuente sistemática de reclutamiento y selección.

- No existe evaluación de rendimiento nl expectativa institucionalizada de permanencia.

- Para el funcionario de carrera, la decisión de aceptar un cargo gerencial representa un riesgo que comprome. te su estabilidad.

- Los cargos no son descritos ni evaluados, por lo que se desconoce su perfil y los requerimientos para su desempeño.

- No se determinan las diferencias de responsabilidad y complejidad entre los cargos; no hay criterios idóneos para asignarles remuneración.

- Se desconocen los factores e indicadores de una buena gestion,

- Los cargos gerenciales están sujetos al llamado "sistema de botin".

- Excesiva individualidad en los gerentes, en perjuicio del trabajo en equipo.

- Predominio de la subjetividad y de la intuición en la toma de decisiones.

- Vulnerabilidad frente al tráfico de influencias.
- Resistencia ante los consejos y recomendaciones de especialistas.

- Temor a la delegación de autoridad hacia sus colaboradores.

- Predominio de expresiones externas de status.

- Deficiente concepción de las prioridades.

- Deficiente capacidad para enfrentar situaciones y problemas.

- Carencia de experiencia.

- Escaso conocimiento de las normas jurídicas que condicionan sus decisiones.

- Limitadas capacidades y habilldades para comprender la conducta humana, asl como para motivar al personal.

- Insuficiente formación en materia de tecnologla administrativa relevante.

- Insuficiente preparación para actuar on un marco de escasez de recursos y para negociar con actores intra y extraorganizacionales.

- Ausencia relativa de un sentido de previsión y de pensamiento estratégico.

\section{Algunas vias de soluclón: la profeslonallzación de la Gerencla Públlca}

En un trabajo desarrollado en el año 1989 por la Comislón Presidencial para la Reforma del Estado (COPRE), se hace énfasis en la profesionalización de la Función Pública como la solución prevista para adaptar ol aparato estatal a las crecientes y múltiples demandas que van diversificando su rol, y adquiere preemi- 
nencia sobre cambios de tipo estructural y procedimental. Se destaca en dicho trabajo que "son la actitud y comportamiento de los funcionarios y de sus gerentes los que van a producir las transformaciones del accionar cotidiano de la Administración". (COPRE, 1989:21)

La experiencia comparada muestra un amplio desarrollo en esta materia. Asi, el informe Northcote Trevelyan de 1853 sirvió de punto de partida para la creación del Civil Service Británico, que fue postenoormente extendido a las colonias. En los Estados Unidos, la Ley Pendleton, origen del sistema de méritos en el Civil Service, data de 1883. La Función Pública francesa recibió primera prioridad recién terminada la Segunda Guerra Mundial. En America Latina, por su parte, ha habido notables avances en la materia, destacándose los casos de Brasil y Argentina.

En Venezuela, la Ley de Carrera Administrativa, promulgada en el año 1971, viabiliza el mandato constitucional. Sin embargo, dicha Ley, en sf misma poco adecuada, es en extremo limitada en cuanto a su ámbito de competencia institucional. Además, no extiende la carrera administrativa a los niveles directivos, de la Administración, los cuales asumen el carácter de botín del partido polltico -0 , en su caso, de la fracción partidista- que triunta en las elecciones presidenciales, con la consecuente ausencia de estabilidad gerencial y de continuidad de los programas gubernamentales de mediano y largo plazo.

Esta última situación ha generado graves disfunciones en la conducción del aparato gubemamental, tales como la imposibilidad de definir horizontes estratégi- cos de largo plazo, corrupción administrativa, subordinación de la maquinaria de gobierno a los intereses de los clanes políticos, etc. (Quintín, 1991:10-15). Ello contrasta con las llamadas "islas de excelencia" que sin duda existen en el sector público. En efecto, aquellos organismos que logran instrumentar una carrera directiva sustentada en el mérito, revelan una gerencia institucional de elevados niveles de excelencia y, por ende, una mayor eficiencia relativa de la organización pública, lo cual incide significativamente en la existencia de un personal con elevados niveles de motivación y de satisfacción en el trabajo, traducióndose en una relación positiva con el ciudadano, en tanto usuario de los servicios de la organización.

En esta perspectiva, la profesionalización persigue justamente eliminar los efectos nocivos que trae consigo el desempeño de funciones gerenciales por parte de personas que llegan a ocupar posiciones directivas sin haber hecho carrera en esas organizaciones, sin haber conocido el complejo mundo de la administración pública, sin haber internalizado los valores derivados de los fines y criterios especfficos del ámbito de la gestión pública; sin conocer las organizaciones y sus funcionarios, y sin entender que la institucionalización que requiere la administración pública exige una continuidad administrativa que se conjugue adecuadamente con la adaptación al entorno turbulento en que se mueve. El objetivo perseguido, en definitiva, consiste en sustituir el sistema de botin imperante por un sistema de carrera, en el entendido de que profesionalizar es crear y aplicar las 
condiciones que permitan hacer y mantener una carrera gerencial püblica.

Podría argumentarse que un cuerpo profesionalizado burocrático puede constituirse en un factor de obstrucción frente a una dirección politica que pretenda lograr transformaciones en el Estado, puesto que tendería al inmovilismo, a la conservación del status, al formalismo y al nitualismo. Sin embargo, ello es más un resultado de la administración de los proyectos y sistemas gubernamentales que de la propia concepción de los modelos de profesionalización gerencial. También en este sentido la experiencia comparada aporta significativas evidencias. En efecto, el desarrollo de los cuerpos de directivos profesionales que emergieron en Europa después de la Segunda Guerra Mundial, se han constituido indistintamente en excelentes apoyos para los gobiernos del más variado signo político. A la inversa, el "sistema del botín", que privó doctrinaria y prácticamente en los Estados Unidos desde el siglo pasado, constituyó el factor desencadenante de la corupción e ineficacia de la Función Pública, y la eliminación de dicho sistema ha sido el aporte más importante de la reforma de la Ley de Servicio Civil en 1978 en ese país.

Hay conciencia, por otra parte, de que la profesionalización de todo el universo de gerentes públicos constituye un proyecto irrealizable hoy en día. Cualquier iniciativa en este sentido requiere de la aceptación política y legislativa por parte de los actores que son beneficiarios actuales o potenciales del "botín" gerencial, quienes, obviamente, no querrán ceder espacios de control burocrático de la Administración ni de prebendas destinadas a compensar la fidelidad a intereses sectoriales ${ }^{3}$.

En tal contexto, la profesionalización de la gerencia pública encuentra viabilidad a través de una estrategia orientada a identificar un conjunto limitado de "cargos críticos", susceptibles de ser sometidos a criterios de reclutamiento, de selección, de estabilidad en función de un sistema de evaluación anual de rendimiento, de remuneraciones competitivas y de formación y reciclaje. Esta estrategia tuvo un éxito significativo en Argentina y en Bolivia; en el primero de estos palses se llegaron a identificar 1.500 cargos criticos, los cuales constituyeron un cuerpo de funcionarios directivos bajo un régimen especial de remuneraciones, diferenciado del resto de la Administración Pública. Para ingresar a dicho cuerpo se exigló aprobar un riguroso sistema de concursos y de credenciales, y someterse a un período de 18 meses de formación en Gerencia Pública. En Bolivia, por su parte, se identificaron 2.200 "cargos claves", en términos similares a la experiencia Argentina.

Ciertamente, la crisis inédita que vive la sociedad venezolana exige, más que en otras coyunturas del pasado, una

La COPAE presentó en el año 1988 un Acuerdo para la Profesionalización de la Gerencia Públlca a ser suscrito por los pertidos polítlcos, el cual no llegó a prosperar. En el año 1994, con asistencla financlera del PNUD, se preparó un Proyecto de Estatuto Gerencial Público que consagrarla la carrera directiva. Este Proyecto será presentado próximamente a la consideración del Elecutivo Naclonal una vez se definan las estrategias pertinentes. 
demanda por administración eficiente de enormes proporciones. Al Estado se le pide, contradictoriamente, que gaste menos, pero simultáneamente se le requiere que haga mucho más de lo que ha hecho tradicionalmente. En el plano político, la demanda por administración eficiente implica la configuración de una administración democrática, que incluya el diseño de mecanismos activos de participación ciudadana, la transparencia de la gestión pública y el control social de la misma.

Una gerencia eficiente en el sector público no se alcanzará sino a partir de un replanteo de las bases conceptuales y de una acción en que el gobiemo y las universidades desempeñen un rol que permita trazar una nueva senda en la conducción de los asuntos públicos.

\section{Conclusiones}

A través de las reflexiones desarrolladas en el presente trabajo, se ha intentado relativizar la importancia -que sin duda la tiene- de las acciones dirigidas a modificar las prácticas gerenciales en la administración pública. No se quiere decir con ello que la Gerencia Pública adquiera un lugar subordinado en la agenda de modemización del sector público. Se trata, más bien, de destacar los factores que permitirlan potenciar los esfuerzos orientados a incrementar la capacidad de gestión del Estado, entre los que se incluyen la formación gerencial.

En tal sentido, es necesario focalizar la reforma hacia aquellos planos que tiendan a configurar una Administración democrática y una relación más abierta y transparente entre el Estado y la socie- dad. Este planteamiento no responde tanto a una sustentación doctrinaria, a priori, sino a un nuevo escenario que progresivamente se ha ido conformando, y que abre paso a actores sociales que tradicionalmente han estado subordinados a los intereses y expectativas de los llamados "clanes políticos". En efecto, liberalizar el mercado trae como consecuencia, en ocasiones imperceptible, la liberalización de las "reglas" que limitan a la sociedad civil y constriñen el juego de la negociación y el conflicto, consustanciales a una forma democrática de organización política.

En este marco, la dirección de los asuntos públicos debe desempeñar un papel absolutamente estratégico y extraordinariamente complejo. Los paradigmas tradicionales de las ciencias gerenclales deben ser sustituidos por nuevos paradigmas que respondan a situaciones reales, turbulentas, poco estructuradas y plenas de incertidumbre. La Gerencia del Sector Público, a su vez, requiere definitivamente ser reconocida en su especiffcidad, como disciplina y como fenómeno social, promoviendo al mismo tiempo una severa transformación de las condiciones ambientales $\theta$ institucionales en que se desenvuelve.

La formación gerencial para el sector público cobra, en esta perspectiva, una importancia de enorme significación. $y$ asi se ha entendido a nivel internacional, particularmente en los palses industrializados. Herramientas analíticas para la planificación, la organización, el análisis de estados financieros, etc.; si bien es cierto son útiles para el desempeño gerencial, y por tanto constituyen materias 
básicas de un programa de estudios en torno a esta área, más importancia tienen aquellos componentes del pensum orientados a desarrollar aspectos tales como: pensamiento estratégico, capacidades técnico-políticas, capacidad para percibir las "señales" de la realidad, capacidad para aprender de las propias experiencias, rigor y consistencia en la investigación organizacional, capacidad para desenvolverse en el tejido social de la organización, orientación a la acción, sustento axiológico del desempeño gerencial, son algunos de los aspectos más sustantivos de la formación de directivos públicos.

Tanto las universidades como el sector público tienen un rol fundamental on torno a las nuevas directrices formativas que se están formulando. Es necesario establecer un "puente de colaboración activa" ente ambas instituciones que permita integrar los escasos recursos disponibles y los esfuerzos que han emprendido diferentes centros de desarrollo de administradores.

\section{Referencias Bibliográficas}

COPRE (1989). La Retorma Adminlstrativa. Caracas.

COPRE (1994). Reiorma de las instituciones de Goblerno. Propuestas para la Modernización del Poder Ejecutivo. Vol. 11. Caracas.

Kotter, John P. (1982). "What effective general managers really do ?". Harvard Business Review. November-december.

Mintzberg, Henry (1975): 'La Alta Direcclón: Mitos y Realidades". Harvard Bussiness Review. Julio-agosto.

Prats i Catala, Joan (1995). "Derecho y Management on las Adminlstraciones Públlcas". Retorma y Democracla. No. 3. CLAD. Caracas.

Quintin, Antonio (1991). Proyecto de creaclón del Insttuto de Alta Gerencla Públlca. COPRE. Mimeo. Caracas.

Senge, Peter (1993). La V Disclpilna. Edlclones Juan Granica S. A. Buenos Aires.

The Economist (1989), July 29. 